\title{
Evaluation of third generation integrated computer network based on principal component analysis method
}

\author{
Yuan BaoLi \\ ShiJiaZhuang Vocational Technology Institute, ShiJiaZhuang, HeBei province , 050081, China \\ stevenybl@qq.com
}

Keywords: PCA methods; 3G Network; CQT Test;network optimization

\begin{abstract}
In this paper, by using the method of comprehensive analysis of principal components, a modeling analysis of test and performance analysis of the network optimization was used. This evaluation system has the characteristics of adaptability, simple application and broad prospects for development.This paper made a detailed introduction to the structure and purpose of network optimization, and its working principle is a simple generalization, concepts and methods of the network performance evaluation and test are described in detail. The principal component analysis method and comprehensive analysis of the evaluation system model, and describes in detail the process and the key points which should be paid attention to in establishing the model of. According to the actual project data of this evaluation model was described and verified the actual, and the results were analyzed and summarized.
\end{abstract}

\section{Introduction}

With the development of wireless network communication services, network optimization plays a very important role, network optimization in a broad sense is a ratio control loop process, through the test and analysis of continuous, adjust the network to reach a high standard requirements and the hardware equipment [1].The second generation mobile communication technology gradually replaced the first generation of mobile communication technology, because the second generation mobile communication system using the digital functions, rather than first generation mobile communication technology has been greatly enhanced, the main characteristics of the second generation mobile communication technology is a high degree of standardization, high security, high utilization of the spectrum [2].

Internet users the technical performance of the network is not concerned about network users care about most is mainly the quality of service of their networks. Simply say that is primarily concerned with network users are connected with the parameters of the quality of the voice, that is, clarity, or download rate and so on $[3,4]$. As managers, the focus of concern in order to pay less for the larger income and therefore managers want to maximize network resources can be utilized, so for managers are most concerned about is the throughput of the network parameters in this context, wood paper proposes principal component analysis and fuzzy comprehensive evaluation combination use to network users and administrators care about the information parameters analyzed together in order to achieve a more comprehensive network performance evaluation judgments, can greatly improve network performance parameters on the current assessment of a single defect $[5,6]$.

In this paper, the principal component analysis and fuzzy comprehensive evaluation method combination, considering the actual network performance evaluation are involved in the various influencing factors KPI indicators presented on a wireless network performance evaluation model, the evaluation method can effectively reflect the CQT test network performance evaluation data obtained in the role, and be able to quickly calculate the required parameters to achieve the desired results envisaged. This paper made a study analyzed the principle of principal component analysis as well as in various fields of application, research and analysis of fuzzy comprehensive analysis of the principle and application in various fields. Study of the principal component analysis and fuzzy comprehensive analysis of combined evaluation model used in the actual situation and the problems involved in the algorithm. 


\section{Evaluation of mobile communication network performance}

The goal of network optimization is now not only been used for wireless network optimization, network optimization services already include user wireless network planning, market forecasting, implementation of the project put into operation until the whole process of each cycle a small part [7]. Excellent access from the technical level there are three objectives, improve customer satisfaction, improve wireless network quality of service and the ability to maintain the stability of wireless network performance. So you want to maintain the stability of wireless network performance will kind of approach to the wireless network, test and analyze operational data, which has played the largest role in the wireless network performance data collection, that is, for its analysis of the data.

Wireless network assessment the operation of the network is through the analysis of the data , objective response network coverage, service performance situation, find the network defects and shortcomings, specifically adjust the target network optimization , network optimization and want to get the initial experience of guiding the wireless network the usual network optimization and maintenance and protection work. Network assessment applies both to assess wireless transmission technology, but also for network services accounted for performance assessment; either as a network pre-construction since the choice of candidate technologies can also be used as network construction, network optimization research into the evaluation after the foundation. The CQT test frame was shown in Fig. 1.

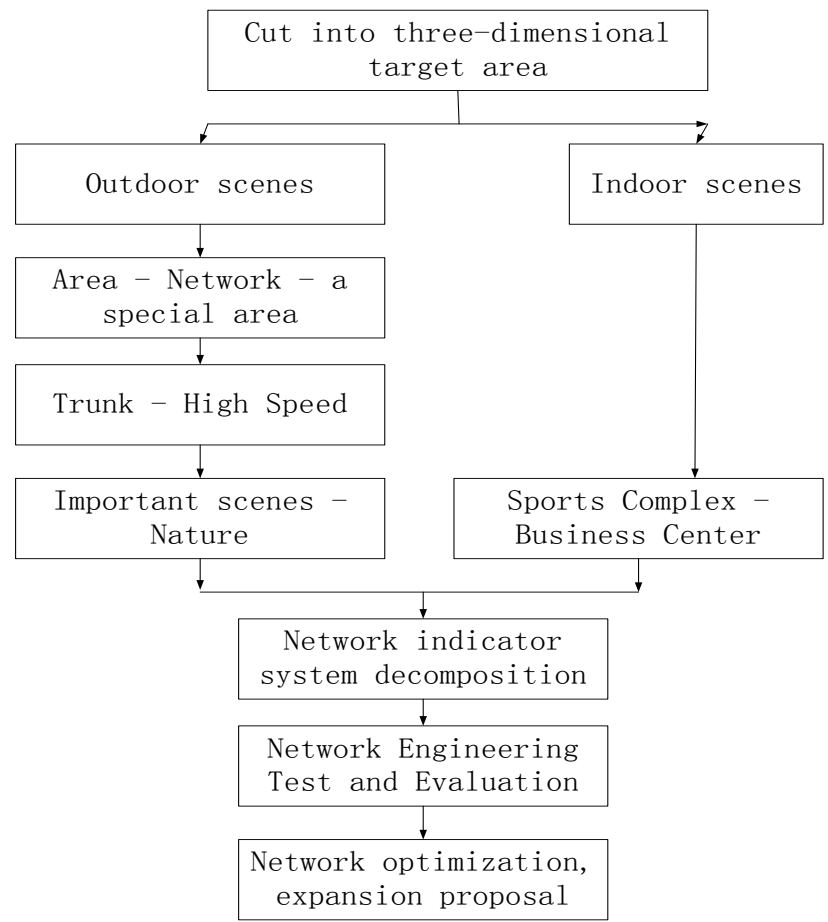

Fig. 1.The CQT test frame

\section{The basic concept of wide area network}

The consist of wide area network. When the distance between the host, for example, tens or hundreds of kilometers apart, or even thousands of kilometers, LAN obviously will not be able to complete the task of communication between hosts. Then you need another structure of the network, the wide area network. WAN switches, and by a number of nodes connected to these switches links. Junction switch performs the packet store and forward capabilities. Point connections between nodes are, but in order to improve the reliability of the network, typically a switch node and multiple nodes are often connected to the switch. Affected by economic conditions, do not use the WAN widely used multiple access technologies. From the level considered, the difference between WAN and LAN great, because the LAN protocol is mainly used for the data link layer (a small amount of the contents of the physical layer), and wide area network protocol used by the network layer. In the WAN is an important issue in routing and packet forwarding. 
WAN is the Internet a core part of its mandate is long transporting host to send data. The nodes connected to the WAN link switches are high-speed links, which may be thousands of kilometers from the fiber-optic cable, it can be tens of thousands of kilometers of point satellite links. Therefore WAN first issue to consider is its communication capacity must be large enough to support the growing traffic. Fig. 2 shows the distant LAN and WAN via a router connected to form a wide range of internet coverage. This can be through the WAN and LAN to another LAN far apart to communicate. A router is a special-purpose host, in Figure two networks will be painted on the outside of it. In fact, it can be simultaneously drawn between the two network, because it is also a wide area network LAN.

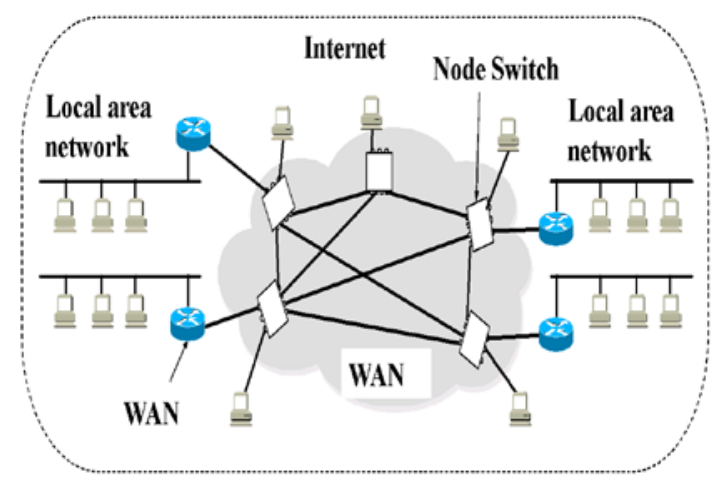

Fig. 2. Internet composed by the LAN and WAN.

Datagram and virtual circuit. From the hierarchical point of view, the top of the WAN is the network layer. Network layer is connected to the network services provided by the host can have two categories, namely connectionless and connection-oriented network services network services. Concrete implementation of these two services is commonly called datagram service and VC services. Fig. 3 are plotted network provides datagram service, and to provide virtual circuit service characteristics. The network layer is the transport layer entity of the user , but for convenience, can be used as the network layer, the host user.

Network provides datagram service is characterized by: the network at all times to accept the host sends a packet. Network for each packet independently selected route. Internet is only best effort to deliver the packet to the destination host, but the network without any commitment on the source host. Network does not guarantee the transmitted packet is not lost, nor guaranteed by the source host sends a packet sequence and within the time limit for how long the packet must be delivered to the destination host. When you need to send the order according to the packet delivered to the destination host, the destination station must also look at the received packet buffer, until the host is able to deliver the order for delivery again. When congestion occurs, a node in the network according to the circumstances of some packet discard.

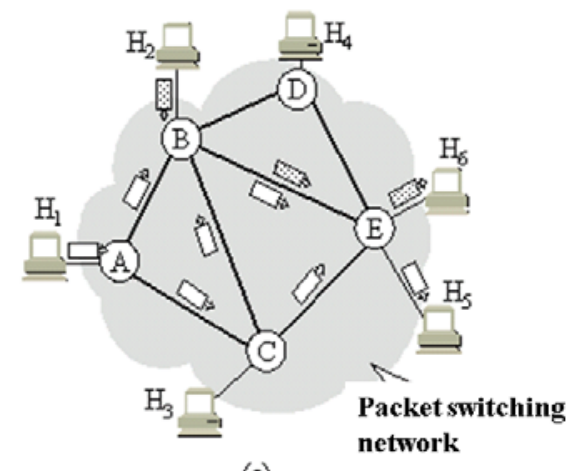

(a)

Fig. 3. Datagram service and virtual circuit services. 


\section{Experimental results}

The reliability of transmission. ARQ protocol works point is in sending a data frame, not stop to wait for an acknowledgment frame, but you can then send a number of consecutive data frames. If you then receive a confirmation sent to the receiving end of the frame, then you can then send the data frame. Due to reduce of waiting times, the communication improves the throughput.

$\mathrm{P}(\mathrm{x})$ is the probability of $\mathrm{X}$ event occurs. So you can get the right to transmit a data frame is the average time required:

$$
\begin{aligned}
& t_{a v}=t_{T}+(1-p) \sum_{i=1}^{\infty} i p^{i} t_{T}=t_{T} /(1-p) \\
& \lambda_{\max }=1 / t_{a v}=(1-p) / t_{T} \\
& \lambda \leq(1-p) / t_{T}(3) \\
& \rho \equiv \lambda t_{f} \leq(1-p) / \alpha<1
\end{aligned}
$$

Continuous ARQ protocol throughput. To easily derived formula continuous ARQ protocol throughput, we assume that there are no errors, the successful transmission of a data frame is the time required $\mathrm{t}_{\mathrm{f}}$. When an error occurs, a retransmission of a data frame is time $\mathrm{t}_{\mathrm{T}}$. Stop waiting for so long as the agreement with reference to formula (7-10), not hard, in the case of continuous ARQ protocol, the correct transmission of a data frame is the average time required:

$$
t_{a v}=t_{f}+(1-p) \sum_{i=1}^{\infty} i p^{i} t_{T}=t_{f}[1+(\alpha-1) p] /(1-p) \text { (5) }
$$

Is saturated at the sending node, the throughput is the maximum:

$\lambda_{\max }=1 / t_{a v}=(1-p) / t_{f}[1+(\alpha-1) p]$

The normalized throughput:

$$
\rho=\lambda t_{f} \leq(1-p) /[1+(\alpha-1) p]
$$

Principal component analysis. Principal component analysis can reduce the indicators analyzed dimension of the space. That use of $\mathrm{m}$-dimensional Y space to replace $\mathrm{P}$-dimensional $\mathrm{X}$ space $(\mathrm{m}<\mathrm{p})$, while the number of low-dimensional space instead of the $\mathrm{Y}$-dimension high $\mathrm{X}$ amount of information lost very little space. That allows only one main component $\mathrm{Y}$ when $\mathrm{Y}$ or application of this $\mathrm{X}$ variable to get. For example, to calculate the average value of $\mathrm{Y}$ when all $\mathrm{X}$ applications still have an average. $M$ out of the front of the selected principal components, the fan if a certain number of $\mathrm{X}$ is approximately 0 , then the $\mathrm{X}$ can be removed, which is a relatively redundant variable exclusion method.

Using principal component analysis hoof pick regression variables. Regression variable choices are pragmatic sense, in order to model the province is easy to do structural analysis, control and forecasting, good original variable from the set consisting of selecting the best variables, constitute the best collection of variables. Using principal component analysis hoof pick variables can be used to select a smaller amount of computation to obtain very sensitive to variable subset selection effect.

We can be found in CQT test a complete occupy relatively large proportion of part or artificial tests, and thus make the test data through the CQT is largely subjective, and thus lack of authenticity, and test each time a test piece so CQT accuracy of the data is also poor. The construction of a large number of base stations and distributed widely enough where wireless communication network characteristics, especially in many more complex terrain or mountainous plateau to $\mathrm{K}$, and therefore in order for these relatively remote and the terrain is more complex areas of a large number of very station construction and coverage is more difficult to test.

Send window is used to control the traffic sender, while the size of the window WT has not received an acknowledgment message, in case the sender can send a maximum number of data frames. Obviously, stop waiting Agreement send window size is 1, indicating that as long as a data frame sent has not been confirmed, you can't send the next data frame. The concept of the send window is best described graphically. 
Let us now send sequence encoded by 3 bits, is sent to eight different serial number, from 0 to 7 . Also assume that the send window $\mathrm{WT}=5$, this means an acknowledgment message is not received in the case, the sender can send out up to five data frames. Send window rules summarized as follows:

(1) Within the window frame transmission is allowed to send a frame, there is no acknowledgment without considering. Send all of the right of the window frames are not allowed to send frames. Figure 8 illustrates this situation.

(2) Each sending a frame, allowing the number of frames sent to minus 1 . However, the position of the transmission window is not changed. Fig. 4 has three different frame: has sent the frame (frame No. 0 leftmost); allowed to transmit a frame (of 4, the left frame 1 to 4 frames), and not transmit a frame (5, frame and the subsequent frames).

(3) If the frame is allowed to be sent are sent over 5, but have not received any confirmation, then no longer send any frames of the. Figure 8 shows this situation. At this time, the sender enters the wait state.

(a)

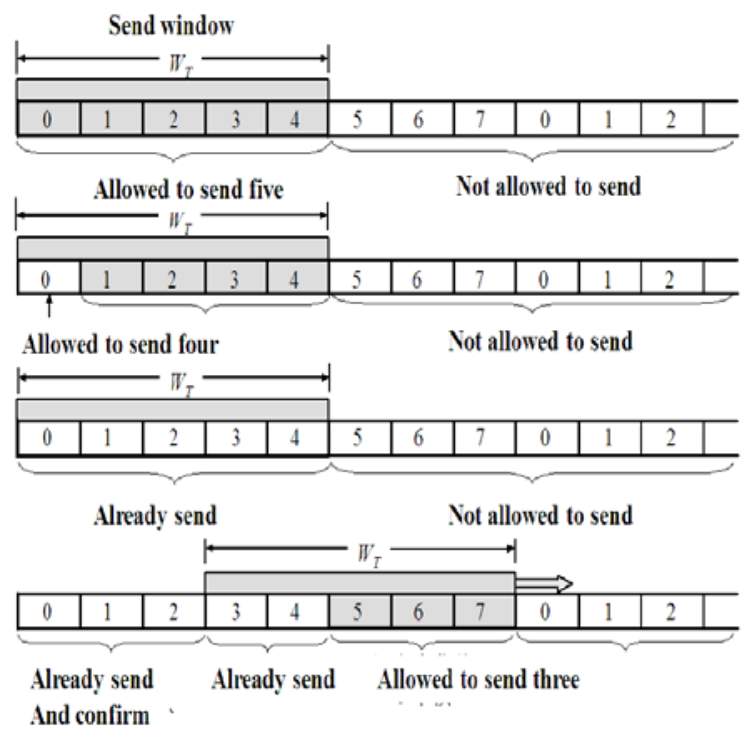

Fig. 4. The transmission window control the transmission rate of the sender.

\section{Summary}

In this paper, a combination of the two evaluation methods used in the network optimization plays a quantification analysis of network quality, network optimization as an important reference and solve problems according to solve the previously Network Optimization the disadvantage of network performance evaluation in a single indicator evaluation methods, while addressing inefficiencies manually compare and subjective evaluation of the shortcomings, thereby improving network quality and network user satisfaction.

\section{References}

[1] A. Tarighat, M. Sadek, A. H. Sayed, “A multi User Beamforming Scheme for Downlink MIMO Channels based on Maximizing Signal-to-Leakage Ratios”, IEEE International Conference on Acoustics, Speech, and Signal Processing, pp. 1129-1132, 2005.

[2] J.van de Beek,O. Edfors, M. Sandell, S. Wilson, P. Borjesson, "On Channel Estimation in OFDM System”, in Proceedings of the IEEE Vehicular Technology Conference, pp. 815-819, 1995.

[3] K.Wong, R. Cheng, K. B. Letaeif, R. D. Murch, "Adaptive antennas at the mobile and base stations in an OFDM/TDMA system”, IEEE Transactions on Communications, vol. 49, no.1, pp. 195-206, 2001. 
[4] M. Sadek, A. Tarighat, A. H. Sayed, "Active Antenna Selection in multi-user MIMO Communications,” IEEE Transactions on Signal Processing, vol. 55, no. 4, pp. 1498-1510, 2007.

[5] Krishnanand K N, Ghose D. Theoretical foundations for rendezvous of glowworm-inspired agent swarms at multiple locations [J]. Robotics and Autonomous Systems, 2008, 56(7): 549-569.

[6] Huang Zhengxin, Zhou Yongquan. Using glowworm swarm optimization algorithm for clustering analysis [J]. Journal of Convergence Information Technology, 2011, 6(2): 78-85.

[7] LI Yong-mei, ZHOU Yong-quan, WEI Jun. Using Hierarchical Structure Glowworm Swarm Algorithm for Function Optimization [J]. JOURNAL OF APPLIED SCIENCES-Electronics and Information Engineering, 2012, 30(4):391-396. 